\title{
Use of homologous costal cartilage in rhinoplasty
}

\author{
Tao Zheng', Yong Ju Jang ${ }^{2}$ \\ 'Department of Otolaryngology Head \& Neck Surgery, Hanyang University Medical Center, College of Medicine, Hanyang University, Seoul \\ 04763, South Korea. \\ ${ }^{2}$ Department of Otolaryngology Head \& Neck Surgery, Asan Medical Center, University of Ulsan, College of Medicine, Seoul 05505, South \\ Korea.
}

Correspondence to: Prof. Yong Ju Jang, Department of Otolaryngology Head \& Neck Surgery, Asan Medical Center, University of Ulsan, College of Medicine, No. 88 Olympic-ro 43-gil, Songpa-gu, Seoul 05505, South Korea. E-mail: 3712yjang@gmail.com

How to cite this article: Zheng T, Jang YJ. Use of homologous costal cartilage in rhinoplasty. Plast Aesthet Res 2019;6:2. http://dx.doi.org/10.20517/2347-9264.2018.76

Received: 5 Nov 2018 First Decision: 24 Nov 2018 Revised: 2 Jan 2019 Accepted: 11 Jan 2019 Published: 24 Jan 2019

Science Editor: Hong Ryul Jin Copy Editor: Cai-Hong Wang Production Editor: Huan-Liang Wu

\begin{abstract}
This review specifically addresses the use of homologous costal cartilage in rhinoplasty with a particular focus on the related complications that can be encountered. It is important to stress that autologous cartilage is probably the preferred material for grafting in rhinoplasty. However, concerns of donor-site morbidity and extensive surgery have motivated the development and use of homologous costal cartilage. Because homologous costal cartilage is readily available, it has been widely used as an alternative to autologous costal cartilage when restoring nasal contour. Both favorable and unfavorable reports can be found in the literature, however, controversy still exists regarding the complications that can occur with using homologous cartilage as a graft material in rhinoplasty. Therefore, the aim of this review is to summarize the current understanding of the usefulness and the problems related with the use of homologous costal cartilage in rhinoplasty.
\end{abstract}

Keywords: Rhinoplasty, homologous cartilage, processed cartilage, irradiated cartilage, preserved cartilage

\section{INTRODUCTION}

In rhinoplasty, surgeons often need ample graft material for septal reconstruction, dorsal augmentation, and tip surgery. Autologous costal cartilage is a preferred source of graft material, especially in Asian patients, as it provides cartilage of enough quantity and quality ${ }^{[1]}$. Theoretically, when it is needed, using autologous costal cartilage is the most ideal surgical strategy. However, autologous costal cartilage may be difficult

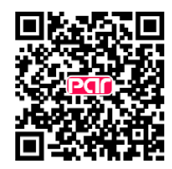


to harvest and may be associated with significant donor site morbidity. Donor site complications such as pneumothorax, seroma, scar-related problems, intercostal neuralgia and intensive postoperative pain are deterring factors for the use of autologous costal cartilage. Increased surgical time can be another concern ${ }^{[2]}$.

In that context, although there can be is limited availability and increased cost for purchasing the graft, the use of homologous costal cartilage in rhinoplasty can be an attractive surgical option as it can avoid donor morbidity and additional operative time. Homologous costal cartilage is harvested from cadaveric donors and is processed in various ways. It has been shown to be useful in rhinoplasty. The tissue processing methods typically involve a high dose of ionizing radiation, osmotic destruction, freeze drying, and chemical sterilization- either alone or in combination- to eliminate the cellularity of the tissue and to sterilize the grafts $^{[3-7]}$. Numerous surgeons have reported their results of using homologous costal cartilage, treated with different processing methods in rhinoplasty. However, controversy still exists in the literature regarding the complications associated with the use of homologous cartilage as a grafting material in rhinoplasty. Therefore, in this paper, we aim to summarize current understanding of the usefulness and the problems related with the use of homologous costal cartilage in rhinoplasty.

\section{REVIEW OF CASE SERIES}

Published reports have mostly dealt with case series of homologous costal cartilage grafts that have been processed in different ways [Table 1]. Mühlbauer et al. ${ }^{[8]}$ reported their experience with the use of L-shaped homologous costal cartilage grafts for saddle nose correction that were preserved in merthiolate saline and stored at $4{ }^{\circ} \mathrm{C}$. Thirty out of the 40 reported cases showed no sign of absorption, moderate absorption was seen in 8 cases and in 2 cases, where syphilis was the underlying pathology, the grafts almost totally disappeared. They believed that the calcification of homologous cartilage began approximately 1 year after insertion and, as time passed, the degree of calcification became more complete and contiguous.

Lefkovits ${ }^{[0]}$ published a retrospective study of 27 augmentation rhinoplasty cases using irradiated homologous costal cartilage (IHCC). The reported complications included infection in 7.4\% (2 of 27) of cases, warping $14.8 \%$ (4 of 27), no resorption was seen. Clark and $\operatorname{Cook}^{[10]}$ studied the usefulness of IHCC in immediate reconstruction of extruded alloplastic nasal implants in 18 patients. They noted that resorption of IHCC was minimal with a mean follow-up of 26 months. There was only one case of warping that was reported and revised by anther IHCC. Strauch and Wallach ${ }^{[11]}$ presented the results of 130 IHCC grafts. There were 2 cases of graft exposure (1.5\%) of immediate complication. Four (3.1\%) cases of late complication, including 1 case of fracture and 2 cases of displacement. There was partial resorption of one graft demonstrated at 6 months postoperatively. Kridel et al. ${ }^{[12]}$ published their experience with the long-term use of HICC in a large series of 357 patients. Among 357 patients, there were 83 primary cases and 274 revision cases. The mean followup period was 13.45 years. The overall complication was $3.25 \%$, including 10 cases of warping (1.06\%), 9 cases of infection (0.86\%), 5 cases of non-infectious resorption (0.53\%), 5 cases of infectious resorption $(0.48 \%), 3$ cases of mobility $(0.31 \%)$ and there was no extrusion noted.

Tutoplast $^{\mathrm{TM}}$ processing is a specific method of tissue processing developed by Tutoplast ${ }^{\mathrm{TM}}$ over forty years ago. The process includes, delipidization, osmotic treatment, oxidative treatment, solvent dehydration, double-sterile packaging, and terminal gamma irradiation (17.8-25.0 kGy). Tutoplast ${ }^{\mathrm{TM}}{ }^{\mathrm{T}}$-processed costal cartilage has very different characteristics than autologous costal cartilage because of its complex chemical process. Demirkan et al. ${ }^{[13]}$ reported their experience with Tutoplast ${ }^{\mathrm{TM}}$-processed homologous costal cartilage use in rhinoplasty in 65 patients, with a mean follow-up period of 33 months. There was no significant resorption detected in any of the cases. However, there were 4 cases $(6 \%)$ of minor complications such as deformity of the dorsal graft, excessive graft length, and erythematous nasal tip. Song et al. ${ }^{[14]}$ studied the surgical outcome of rhinoplasty using Tutoplast ${ }^{\mathrm{TM}}$-processed costal cartilage. They reported a relatively high complications rate of $31 \%$. These included 6 (17\%) cases of partial resorption, $3(9 \%)$ cases of warping, 1 (3\%) 
Table 1. A summary of study characteristics

\begin{tabular}{|c|c|c|c|}
\hline References & Processing type & Complications & Follow-up period mean (range) \\
\hline $\begin{array}{l}\text { Mühlbauer et al. }{ }^{[8]}, 1971 \\
(n=40)\end{array}$ & Merthiolate & $\begin{array}{l}\text { Absorption, } 25 \% \\
\text { Warping, 2.5\% } \\
\text { Late infection, 0\% }\end{array}$ & $\begin{array}{l}\text { Mean, } 6 \text { years } \\
\text { (range, } 1 \text { month-10 years) }\end{array}$ \\
\hline $\begin{array}{l}\text { Lefkovits }^{[9]}, 1990 \\
(n=24)\end{array}$ & Irradiation & $\begin{array}{l}\text { Absorption, } 0 \% \\
\text { Warping, } 14.8 \% \\
\text { Infection, } 7.4 \%\end{array}$ & (range, 1-27 months) \\
\hline $\begin{array}{l}\text { Clark and Cook }{ }^{[10]}, 2002 \\
(n=18)\end{array}$ & Irradiation & $\begin{array}{l}\text { Warping, 5.6\% } \\
\text { Infection, } 0 \% \\
\text { Extrusion, 0\% }\end{array}$ & Mean, 26 months \\
\hline $\begin{array}{l}\text { Strauch and Wallach }{ }^{[11]}, 2003 \\
(n=51)\end{array}$ & Irradiation & $\begin{array}{l}\text { Extrusion, } 1.5 \% \\
\text { Displacement, 1.5\% } \\
\text { Absorption, } 0.8 \% \\
\text { Fracture, } 0.8 \% \\
\text { Infection, } 0 \% \\
\text { Warping, 0\% }\end{array}$ & (range, 7 months -12 years) \\
\hline $\begin{array}{l}\text { Song et al. }{ }^{[14]}, 2008 \\
(n=35)\end{array}$ & Tutoplast & $\begin{array}{l}\text { Resorption, 17\% } \\
\text { Warping, 9\% } \\
\text { Visible contour, 3\% } \\
\text { Frature, 3\% } \\
\text { Infection, 0\% }\end{array}$ & $\begin{array}{l}\text { Mean, } 15.6 \text { months } \\
\text { (range, } 9-35 \text { months) }\end{array}$ \\
\hline $\begin{array}{l}\text { Kridel et } a l^{[12]}, 2009 \\
(n=357)\end{array}$ & Irradiation & $\begin{array}{l}\text { Warping, } 1.06 \% \\
\text { Noninfection resorption, } 0.53 \% \\
\text { Infection resorption, } 0.48 \% \\
\text { Infection, } 0.87 \% \\
\text { Mobility, } 0.31 \% \\
\text { Extrusion, } 0 \%\end{array}$ & $\begin{array}{l}\text { Mean, } 13.45 \text { years } \\
\text { (range, } 4 \text { days- } 24 \text { years) }\end{array}$ \\
\hline $\begin{array}{l}\text { Suh et } a{ }^{[15]}, 2013 \\
(n=30)\end{array}$ & $\begin{array}{l}\text { Tutoplast } \\
\text { Allowash }\end{array}$ & $\begin{array}{l}\text { Fracture, } 6.7 \% \\
\text { Nasal obstruction, 3.3\% } \\
\text { Resorption, 0\% } \\
\text { Warping, 0\% } \\
\text { Extrusion, 0\% } \\
\text { Tip stiffness, } 73.3 \%\end{array}$ & Mean, 29 months \\
\hline
\end{tabular}

graft fracture and 1 (3\%) visible graft contour [Figure 1]. Lohuis et al. ${ }^{[15]}$ used diced Tutoplast ${ }^{\mathrm{TM}}$ homologous cartilage for dorsal augmentation. Their 9 patients showed no complications during the follow-up period of 20 months.

Homologous costal cartilage was mostly used for dorsal augmentation, but it can serve as a useful grafting material for septal reconstruction. Suh et al. ${ }^{[16]}$ reported their 2-year follow-up data in 30 cases on the use of irradiated homologous costal cartilage as a septal extension graft for the correction of the contracted nose in Asians. There were 2 cases of graft fracture observed. Three patients underwent revision rhinoplasty, one for nasal obstruction and 2 because of dissatisfaction with the shape of the nasal tip.

Our review of these case series indicates that Tutoplast ${ }^{\mathrm{TM}}$-processing methods have higher complication rate than IHCC $^{[14]}$. This observation has already been confirmed by a systematic review by Lee et al. ${ }^{[17]}$ As described in the literature, the use of homologous costal cartilage in rhinoplasty has yielded conflicting results regarding the rate of resorption and warping. Although it is difficult to directly compare the surgical outcomes of all the reports because of the differences in follow-up period and processing methods of the cartilage, the rate of complications including resorption, warping, fracture, and infection varies greatly between different studies. The factors responsible for increased graft resorption and warping are not clear, but they may include the size and site of the implant, the carving technique of the graft, the nature of the host recipient site, and thermal and mechanical damage caused during carving. The degree of warping may also depend on the amount of radiation to which the cartilage has been exposed ${ }^{[18]}$. Although, Adams et al. ${ }^{[19]}$ showed that there was no significant difference in warping between irradiated and nonirradiated homologous cartilage in vitro, it is generally believed that the radiation dose has some impact on warping. Donald et al. ${ }^{[18]}$ postulated that the factor governing different absorption rates of IHCC from different studies might be the radiation dose. And the study reported that high-dose irradiation appears to 


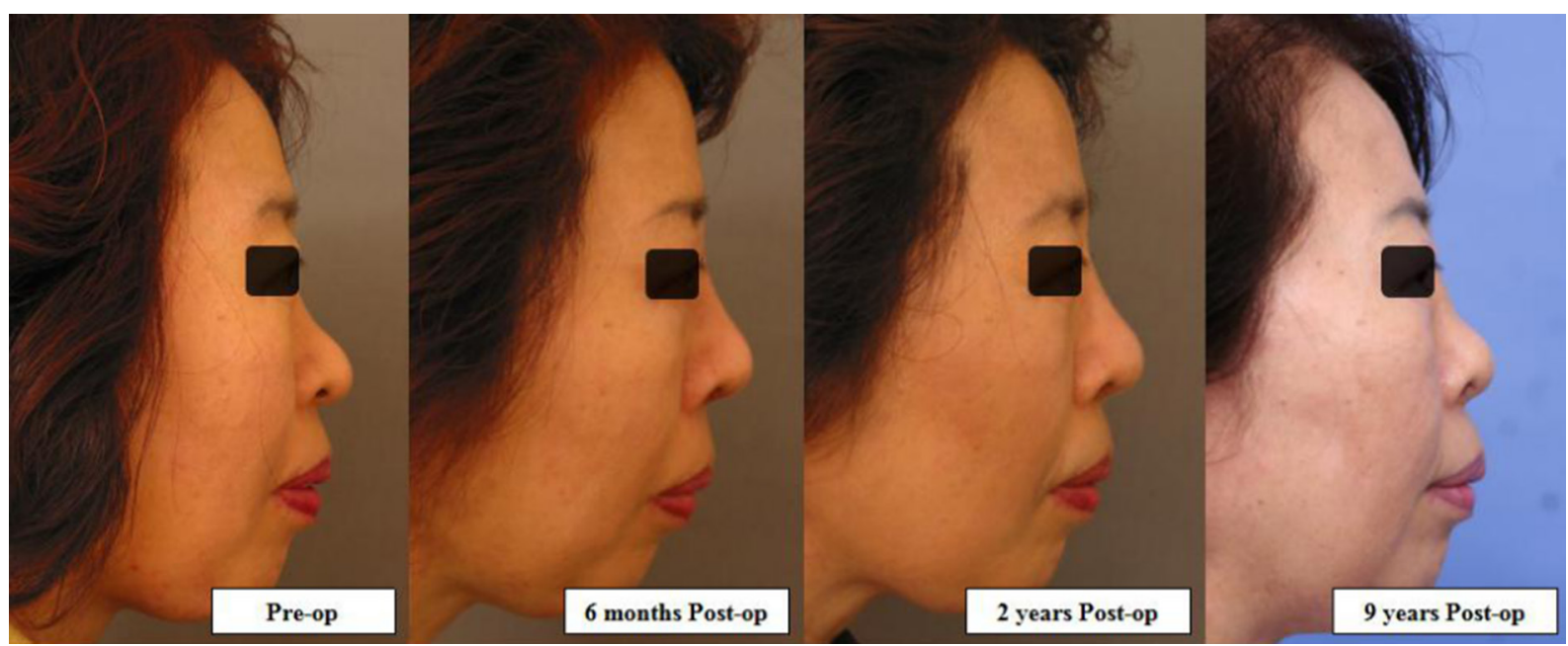

Figure 1. Surgical outcome of rhinoplasty using Tutoplast-processed costal cartilage

lessen greatly the stiffness of cartilage grafts and may be responsible for increasing absorption of the graft. Not only the tissue processing method, but also a consistent and meticulous way of tissue handling may be an important factor in reducing complication rates. Kridel and Sturm ${ }^{[20]}$ recommended that sterile handling, followed by a rinse with saline and antibiotics, removal of perichondrium, waiting $20 \mathrm{~min}$ after carving to allow time for initial warping, and the use of perioperative antibiotics are important elements to reduce complications.

The fate of implanted homologous cartilage has been studied. Suh et al. ${ }^{[16]}$ took biopsies of the irradiated cartilage grafts at 18 months and 5 years postoperatively from the study group were compared with preoperative IHCC samples and normal rib cartilage from other patients. They noted that the normal rib cartilage showed intact chondrocytes with extensive HLA-B expression, whereas the preoperative IHCC samples showed no visible normal chondrocytes despite well-maintained lacunar structures, and with much less HLA-B antigen expression. They also found the formation of the thin capsule-like tissue surrounding the IHCC.

It is noteworthy to mention that other than the commonly noted complications of homologous costal cartilage, the possibility of transmission of unknown viral or prion disease which is difficult to screen can be a potential concern when we consider the use of homologous costal cartilage in rhinoplasty. However, studies are lacking on this subject. Freeze drying is a different method of tissue processing, but there is a paucity of studies showing the performance of homologous costal cartilage processed with this particular technique.

\section{CONCLUSION}

Previous reports frequently indicate that homologous cartilage is often more easily absorbed than autologous cartilage, and the senior author has had a similar experience. Furthermore, since cartilage differs in physical characteristics based on tissue processing methods and the cadavers from which the cartilage is harvested are of different age groups, it is hard to expect a consistent quality of cartilage from this implant. The authors have abundant experience in rhinoplasty using Tutoplast ${ }^{\mathrm{TM}}$ processed costal cartilage ${ }^{[14]}$. According to the author's experience, this cartilage is generally useful for septal reconstruction or tip surgery. However, when used for the nasal dorsum, complications such as resorption, fracture, and deformation are somewhat frequent. Therefore, we came to the conclusion that it is not the optimal material to be used for dorsal grafting. However, this graft is still a useful alternative for patients that require ample graft material, where 
the harvest of autologous costal cartilage is a problem. Patients who do not want a donor site scar and older patients that may have extensive ossification of costal cartilage can be ideal candidates for the use of homologous costal cartilage.

\section{DECLARATIONS}

\section{Authors' contributions}

Conception of the study and performed data analysis and interpretation, involved in drafting and revising the manuscript: Jang YJ

Performed data acquisition and drafting the manuscript: Zheng $\mathrm{T}$

\section{Availability of data and materials}

Not applicable.

\section{Financial support and sponsorship}

None.

\section{Conflicts of interest}

Both authors declared that there are no conflicts of interest.

\section{Ethical approval and consent to participate}

Not applicable.

\section{Consent for publication}

Not applicable.

\section{Copyright}

(C) The Author(s) 2019.

\section{REFERENCES}

1. Gunter JP, Cochran CS, Marin VP. Dorsal augmentation with autogenous rib cartilage. Semin Plast Surg 2008;22:74-89.

2. Varadharajan K, Sethukumar P, Anwar M, Patel K. Complications associated with the use of autologous costal cartilage in rhinoplasty: a systematic review. Aesthet Surg J 2015;35:644-52.

3. Rasi HB. The fate of preserved human cartilage. Plast Reconstr Surg 1959;24:24-33.

4. Dingman RO, Grabb WC. Costal cartilage homografts preserved by irradiation. Plast Reconstr Surg 1961;28:562-7.

5. O'Connor GB, Pierce GW. Refrigerated cartilage isografts. Surg Gynecol Obstet 1938;67:796-8.

6. Peer LA. Transplantation of Tissues. Vol 1. Baltimore, MD: William and Wikins Co; 1955.

7. Asbury RB, Dingman R, Lynch JD. The effectiveness of sterilization of canine costal cartilage by cobalt60 irradiation and its fate when used in homografts. Surg Forum 1956;6:581-5.

8. Mühlbauer WD, Schmidt-Tintemann U, Glaser M. Long-term behaviour of preserved homologous rib cartilage in the correction of saddle nose deformity. Br J Plast Surg 1971;24:325-33.

9. Lefkovits G. Irradiated homologous costal cartilage for augmentation rhinoplasty. Ann Plast Surg 1990;25:317-27.

10. Clark JM, Cook TA. Immediate reconstruction of extruded alloplastic nasal implants with irradiated homograft costal cartilage. Laryngoscope 2002;112:968-74.

11. Strauch B, Wallach SG. Reconstruction with irradiated homograft costal cartilage. Plast Reconstr Surg 2003;111:2405-11; discussion 2412-3.

12. Kridel RW, Ashoori F, Liu ES, Hart CG. Long-term use and follow-up of irradiated homologous costal cartilage grafts in the nose. Arch Facial Plast Surg 2009;11:378-94.

13. Demirkan F, Arslan E, Unal S, Aksoy A. Irradiated homologous costal cartilage: versatile grafting material for rhinoplasty. Aesthetic Plast Surg 2003;27:213-20.

14. Song HM, Lee BJ, Jang YJ. Processed costal cartilage homograft in rhinoplasty: the Asan Medical Center experience. Arch Otolaryngol Head Neck Surg 2008;134:485-9.

15. Lohuis PJ, Joshi A, Bran GM, Datema FR, Vermeeren L. Dorsal onlay with diced homologous processed rib cartilage grafts. Aesthetic Plast Surg 2017;41:140-5. 
16. Suh MK, Ahn ES, Kim HR, Dhong ES. A 2-year follow-up of irradiated homologous costal cartilage used as a septal extension graft for the correction of contracted nose in Asians. Ann Plast Surg 2013;71:45-9.

17. Lee MR, Unger JG, Rohrich RJ. Management of the nasal dorsum in rhinoplasty: a systematic review of the literature regarding technique, outcomes, and complications. Plast Reconstr Surg 2011;128:538-50e.

18. Donald PJ, Deckard-Janatpour K, Sharkey N, Lagunas-Solar M. The effects of irradiation dose on the stiffness of cartilage grafts. Ann Plast Surg 1996;36:297-303.

19. Adams WP Jr, Rohrich RJ, Gunter JP, Clark CP, Robinson JB Jr. The rate of warping in irradiated and nonirradiated homograft rib cartilage: a controlled comparison and clinical implications. Plast Reconstr Surg 1999;103:265-70.

20. Kridel RW, Sturm AK. Dorsal Augmentation with Homologous Rib. Facial Plast Surg 2017;33:195-201. 